Universidad de Lima

Facultad de Psicología

Carrera de Psicología

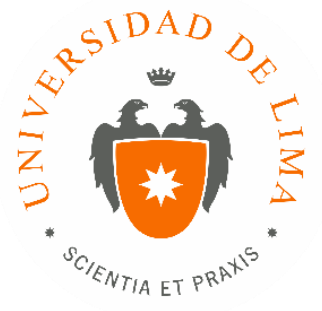

\title{
EXPERIENCIA DE TRABAJO EN UN PROYECTO SOCIAL DE ATENCIÓN PSICOTERAPÉUTICA GRATUITA
}

Trabajo de suficiencia profesional para optar el título profesional de Licenciado en Psicología

\section{Carlos Alonso Miranda Passalacqua}

Código: 20080664

Lima - Perú

Febrero de 2019 



\section{EXPERIENCIA DE TRABAJO EN UN PROYECTO SOCIAL DE ATENCIÓN PSICOTERAPÉUTICA GRATUITA}




\section{TABLA DE CONTENIDO}

INTRODUCCIÓN

CAPÍTULO I: IDENTIFICACIÓN DEL PROBLEMA

CAPÍTULO II: DESCRIPCIÓN DE LAS ACTIVIDADES REALIZADAS

2.1 Promoción de salud psicosocial intra y extra muros 10

2.1.1 Taller dirigido a padres sobre orientación para la crianza 11

2.1.2 Taller para padres y madres sobre violencia familiar 11

2.1.3 Taller de prevención de adicciones y conductas sexuales de riesgo para los alumnos de un colegio no escolarizado 12

2.1.4 Charla sobre orientación vocacional en un colegio público 12

2.2 Intervenciones individuales 13

2.2.1 Psicoterapia 13

2.2.2 Evaluación y diagnóstico psicológico 13

2.3 Propuestas y ejecución de los procedimientos de atención 14

2.3.1 Informes de evolución clínica 14

2.3.2 Propuesta 1: Encuesta de satisfacción terapéutica 14

2.3.3 Propuesta 2: Recomendaciones generales 14

\section{CAPÍTULO III: RESULTADOS DE LA INTERVENCIÓN}

3.1 Talleres para padres (orientación para la crianza y violencia familiar) 15

3.2 Taller y charla para alumnos (adicciones, educación sexual y orientación vocacional)

3.3 Psicoterapia, evaluación y diagnóstico psicológico 16 


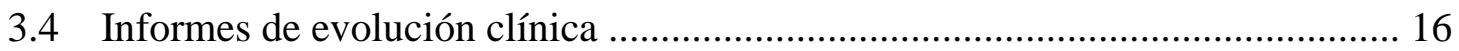

3.5 Propuestas para los procedimientos de atención ............................................ 16

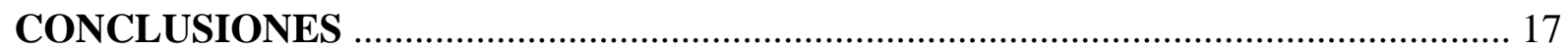

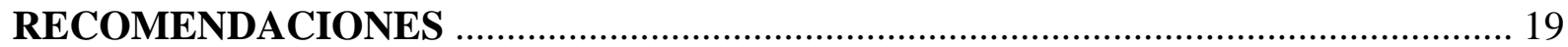

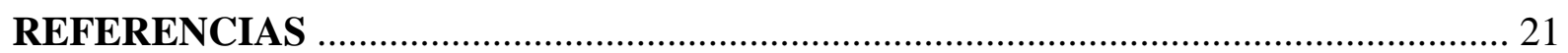

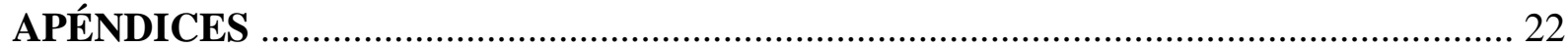




\section{ÍNDICE DE APÉNDICES}

Apéndice 1: Encuesta de satisfacción terapéutica

Apéndice 2: Recomendaciones generales 


\section{INTRODUCCIÓN}

A partir del año 1995, se conformó el proyecto social aludido en el título del presente trabajo; que consiste hasta hoy en día, en brindar servicios de atención psicológica gratuitos a los pobladores de un distrito limeño de bajos recursos socio-económicos. Más adelante, a partir de 1999, el Proyecto empezó a recibir financiación de una universidad europea.

Esta iniciativa cobra especial relevancia tanto por la falta de profesionales de salud mental que existe en el Perú, en contraste con las necesidades de la población y por los insuficientes presupuestos estatales dedicados a éstas (Alarcón, 2015); como por las características de la población atendida en el Proyecto (vecinos de un distrito de escasos recursos): personas expuestas a altos índices de delincuencia y de consumo de sustancias, a la pobreza, la marginalidad, bajos niveles socioculturales y mayor incidencia de violencia familiar. Factores de riesgo para desarrollar una amplia diversidad de psicopatologías duraderas y de alta severidad (Alarcón, 2015; Del Águila-Escobedo, 2015 y Salas, 2018).

Mediante el Proyecto, se intenta cubrir ese gran vacío de promoción de la salud psicosocial de los peruanos, con talleres que abordan temas de capital importancia tanto por incidir de manera decididamente negativa en la dimensión psicológica de las personas; como por ser moneda corriente dentro de las poblaciones vulnerables. Se hace referencia a aspectos como la violencia familiar, el maltrato (físico y psicológico) infantil, las adicciones, entre otros. Asimismo, se atiende en psicoterapia individual a niños, adolescentes, adultos y adultos mayores, amortiguando los efectos de los estresores circundantes, previniendo el desarrollo de futuros trastornos mentales y procurando mejorar la calidad de vida de la población local. 


\section{CAPÍTULO I: IDENTIFICACIÓN DEL PROBLEMA}

Existe en el Perú por un lado, escasez de profesionales de la salud mental en contraste con los requerimientos y necesidades de la población, y por otro, presupuestos nacionales, regionales y locales insuficientes para tales fines (Alarcón, 2015).

En ese contexto, a partir del año 1995, un grupo de psicólogos y estudiantes de psicología conformaron el proyecto social antemencionado; que consiste hasta hoy en día, en brindar servicios de atención psicológica gratuitos a los pobladores de un distrito limeño de bajos recursos socioeconómicos. Más adelante, a partir de 1999, el Proyecto empezó a recibir financiación de una universidad europea; la cual envía anualmente practicantes preprofesionales que apoyan en todos los servicios, programas e intervenciones que se realizan a través del Proyecto.

Cabe mencionar que la gran mayoría de usuarios de los servicios brindados, son personas socioeconómicamente desfavorecidas; que usualmente viven en condiciones precarias y su nivel de instrucción (en el caso de los adultos) no suele superar la secundaria. Asimismo, en el distrito en cuestión la violencia, el consumo de alcohol y drogas, el trabajo infantil, los bajos niveles socio-culturales, la pobreza y el desempleo; son más frecuentes que en otros lugares, en razón de la exclusión social y la falta de desarrollo económico y cultural que allí se observa.

Lo dicho en el párrafo anterior configura una amplia gama de factores de riesgo para desarrollar psicopatologías crónicas de alta severidad, conductas disfuncionales, desadaptación, enfermedades físicas, pobre rendimiento intelectual, conductas de riesgo (drogadicción, conductas sexuales inseguras, mala alimentación, deserción escolar, etc.), familias violentas y disfuncionales, baja autoestima e hipersensibilidad al estrés (Alarcón, 
2015; Del Águila-Escobedo, 2015 y Salas, 2018). Todo lo cual acaba redundando en una dificultad extrema para el progreso socio-económico personal (pues ello implica estar capacitado emocional e intelectualmente para iniciar y terminar una carrera y conseguir y mantenerse en un trabajo o diseñar un proyecto empresarial lucrativo) y en mayores gastos públicos en áreas como salud, justicia y educación.

Otras dos características de la población con las que tienen que lidiar los miembros del equipo del Proyecto son, por un lado, que aquélla presenta en su mayoría aptitudes de expresión y comprensión verbal bastante limitadas, un pensamiento más bien concreto y por consiguiente, dificultad para entender metáforas, diferenciar sus propios pensamientos de sus emociones, entender las causas de sus reacciones y efectuar correctamente diversas técnicas cognitivas.

Por otro lado, una gran cantidad de usuarios de psicoterapia individual, asisten únicamente a una sesión, y otros tantos a un máximo de tres sesiones. Es posible que tal fenómeno se deba a que al estar tan ocupados en "ganarse la vida", carecen de tiempo suficiente para asistir con regularidad. También se considera la hipótesis de que al ser gratuito el servicio, no le otorgan gran valor, creyendo que pueden regresar en cualquier momento y, por último, que quienes desertan tenían expectativas diferentes de en qué consiste una sesión de psicoterapia y creían que en poco tiempo, de alguna forma milagrosa, iban a poder superar sus dificultades emocionales con ayuda de su consejero/terapeuta. Es factible que ocurra una combinación de los factores mencionados. 


\section{CAPÍTULO II: DESCRIPCIÓN DE LAS ACTIVIDADES Y TAREAS REALIZADAS}

\subsection{Promoción de salud psicosocial intra y extra muros}

Se ofrecieron talleres y dictaron charlas sobre las siguientes temáticas (tal como se describe más adelante en cada división del presente subtítulo): orientación para la crianza, violencia familiar, adicciones, educación sexual y orientación vocacional. De manera sucinta se señala la relevancia de las temáticas. La mayor incidencia de violencia infantil se produce en lugares de gran pobreza, exclusión social y bajo nivel educativo (Del Águila-Escobedo, 2015). Este hecho verificado, se conjuga con que la agresividad del agresor que se descarga contra su pareja o hijos, casi en todos los casos, es consecuencia, en gran parte, de las frustraciones que sobrevienen a castigos físicos, maltratos psicológicos, privaciones y marginación sufridos durante la infancia o la niñez (Miljánovich et al., 2014).

Por otro lado, las intervenciones relacionadas con las adicciones y la educación sexual, tuvieron como finalidad prevenir en adolescentes, desenlaces a la vez trágicos y tempranos en sus vidas, ya sea por contraer una infección de transmisión sexual (ITS) que reduzca su calidad o esperanza de vida, por exponerse a un aborto o a tener que cuidar y mantener a un hijo no deseado, o por tener que luchar para siempre contra alguna adicción. Tómese en cuenta que, es la etapa de la adolescencia un terreno fértil para que germinen las conductas inseguras en virtud de la avidez de los adolescentes por experimentar, explorar y asumir riesgos en pro de alcanzar autonomía, destacar entre sus pares y construir su identidad (Salas, 2018). 
Finalmente, con respecto a la orientación vocacional, se buscó llevar a cabo lo que preconizan Sánchez et al. (2017), esto es, ayudar a los jóvenes a tomar decisiones vocacionales acordes tanto a su potencial, cuanto a su proyecto de vida y en concordancia con la oferta educativa y laboral actual; en la misma línea con lo propuesto por Echevarría y Martínez (2014), quienes señalan que un resultado importante de la orientación vocacional ha de ser que se reduzca el número de personas que no logran la inserción laboral, por no responder a los requerimientos ocupacionales o haberse desentendido de sus posibilidades reales de empleabilidad al finalizar su formación.

\subsubsection{Taller dirigido a padres sobre orientación para la crianza}

Se desarrolló con apoyo de material audiovisual didáctico, se contestaron inquietudes y se formularon preguntas y situaciones problemáticas a los asistentes para verificar su comprensión sobre lo explicado. Los objetivos fueron: concientizar sobre la importancia de evitar la violencia física y psicológica en la crianza, explicar nociones básicas de condicionamiento clásico y operante, proponer la extinción de las conductas problema mediante el castigo negativo, incentivar la validación emocional de los hijos, y establecer las diferenciaciones entre conducta asertiva, pasiva y agresiva.

\subsubsection{Taller para padres y madres sobre violencia familiar}

Se impartió con apoyo de material audiovisual didáctico, se contestaron preguntas, se formularon preguntas y situaciones problemáticas para verificar la comprensión de lo explicado, se hicieron demostraciones de 
"role-playing” y dinámicas para demostrar las habilidades aprendidas. Los objetivos fueron: demostrar el ciclo de la violencia, explicar la escalera de la violencia y analizar y modificar creencias y expectativas disfuncionales de violencia familiar.

\subsubsection{Taller de prevención de adicciones y conductas sexuales de riesgo para} los alumnos de un colegio no escolarizado

Respecto de las adicciones se trataron las temáticas de: 1) qué es una adicción y cómo detectarla, 2) causas de las adicciones, 3) consecuencias de las adicciones comunes: marihuana, alcohol, clorhidrato de cocaína, pasta básica de cocaína, casinos, redes sociales y videojuegos, y 4) motivación para no caer en adicciones: dinero ahorrado, deporte, vida larga, relaciones de pareja satisfactorias, futuro profesional, salud, estabilidad emocional, etc. En cuanto a la educación sexual se habló de: 1) la importancia de que toda relación sea consentida y sin dañar a terceros, 2) uso de anticonceptivos, 3) métodos para prevenir ITS, 4) consecuencias de las ITS, y 5) consecuencias de los embarazos no deseados.

\subsubsection{Charla sobre orientación vocacional en un colegio público}

Se desarrolló con ayuda de material audiovisual y se respondieron preguntas acerca de: 1) cómo saber qué se enseña en la profesión que quiero estudiar, 2) cómo saber qué hacen los profesionales de la profesión que quiero seguir, 3) dónde puedo estudiar, 4) qué carreras técnicas y profesionales son más demandadas actualmente, y 5) qué ayudas ofrece el Estado. 


\subsection{Intervenciones individuales}

Fue la actividad principal y la que más tiempo demandó. Se realizaron intervenciones individuales con niños, adolescentes, adultos y adultos mayores. Asimismo, se atendieron parejas y se efectuaron consejos para la crianza a padres que solicitaban atención para sus hijos menores.

\subsubsection{Psicoterapia}

El esqueleto teórico de las sesiones de psicoterapia fue el de la corriente cognitivo-conductista, de verificada eficacia (Camacho, 2014); sin embargo, en la medida en que los pacientes presentaban una capacidad de pensamiento abstracto limitada y una alta necesidad de direccionalidad, se puso énfasis en las técnicas netamente conductistas. A contrario sensu, en los casos de pacientes con una inteligencia balanceada, se aplicaron incluso técnicas propias de las terapias contextuales tales como las descritas por Pérez (2014): mindfulness, defusión cognitiva, aceptación radical (en oposición a la evitación experiencial), etc. Todo ello en pro del respeto por las técnicas e intervenciones cuya efectividad esté basada en la evidencia. Esto es, en armonía con el rigor científico.

\subsubsection{Evaluación y diagnóstico psicológico}

La evaluación psicológica se hizo principalmente a través de la entrevista terapéutica. No en todos los casos de consulta se pudo establecer un diagnóstico, pero sí se logró delimitar los síntomas (cognitivos, afectivos, conductuales y fisiológicos) y los objetivos terapéuticos. 


\subsection{Propuestas y ejecución de los procedimientos de atención}

Se ejecutaron oportunamente los procedimientos de atención establecidos por el Proyecto y se hicieron además dos propuestas.

\subsubsection{Informes de evolución clínica}

Se registraron en fichas: datos recabados durante sesiones, resultados de las pruebas tomadas, técnicas utilizadas, tareas asignadas, la reducción de síntomas si fuera el caso, y la planificación de futuras estrategias de intervención (con base en el repaso teórico o consulta con los supervisores).

\subsubsection{Propuesta 1: Encuesta de satisfacción terapéutica}

Dado que, una considerable cantidad de los pacientes no regresaba a consulta después de la primera sesión, quien esto escribe tomó a bien diseñar una encuesta de satisfacción terapéutica (ver apéndice 1), para verificar si el paciente se encontraba satisfecho con el servicio recibido. Consistía en cuatro preguntas que el paciente debía contestar a solas y de forma anónima. Luego, otro miembro del equipo distinto a quien abordaba el caso, debía recoger la hoja y colocarla en una caja cerrada.

\subsubsection{Propuesta 2: Recomendaciones generales}

Semanas antes de finalizar el año de trabajo, el autor de este texto redactó y envió al coordinador del Proyecto una serie de recomendaciones para hacer más efectivo el servicio brindado (ver apéndice 2), en concordancia con los problemas identificados. 


\section{CAPÍTULO III: RESULTADOS DE LA INTERVENCIÓN}

\subsection{Talleres para padres de familia (orientación para la crianza y violencia}

familiar)

Los resultados fueron la realización misma de los talleres, que contaron con 6 sesiones (orientación para la crianza) y 4 sesiones (violencia familiar) respectivamente. La realización de los talleres fue comunicada a la comunidad mediante pancartas y también directamente a los pacientes con motivos de consulta relacionados con los temas a tratar. Se observó un efecto de "radio bemba", pues en cada sesión nueva siempre hubo más asistentes que en la anterior y, personas que no conocían del servicio de psicoterapia brindado por el Proyecto, que se enteraron de éste a través de los talleres, empezaron a acudir a psicoterapia individual. Por último, cabe destacar que, aunque la mayoría de asistentes demostraron comprender los mensajes transmitidos, muchos de ellos consideraban normal e incluso adecuado el uso de violencia física como modo de "corregir" a sus hijos o enfrentarse a sus parejas.

\subsection{Taller y charla para alumnos (adicciones, educación sexual y orientación} vocacional)

Los resultados incluyen la realización misma del taller y la charla, que contaron con 4 sesiones y 1 sesión respectivamente. Desafortunadamente, los alumnos muchas veces no supieron responder a las preguntas que se les planteó para corroborar que habían entendido lo explicado. Asimismo, hubo que llamarles reiteradamente la atención, para que dejasen de conversar y presten atención. Se 
asume que esto se debe en gran parte a su falta de convencimiento respecto de la utilidad de la información proporcionada.

\subsection{Psicoterapia, evaluación y diagnóstico psicológico}

Los resultados incluyen la atención de 66 pacientes; entre niños, adolescentes, adultos y adultos mayores, en 384 sesiones. En cada caso se estableció o bien un diagnóstico multiaxial o bien la delimitación de los síntomas (afectivos, cognitivos, conductuales y fisiológicos) de acuerdo al motivo de consulta. Éstos fueron evaluados principalmente mediante la entrevista clínica y eventualmente con algún test psicológico.

\subsection{Informes de evolución clínica}

El resultado fue la elaboración de un informe de evolución por cada paciente (66 en total), debidamente archivado en el folder del período de atención, que sirvió de guía y apoyo a los siguientes terapeutas/consejeros que abordaron los casos de los pacientes que continuaron asistiendo a psicoterapia o que retomaron las sesiones después de un tiempo de inasistencia.

\subsection{Propuestas para los procedimientos de atención}

Se diseñó una encuesta de satisfacción terapéutica (ver apéndice 1), con el fin de determinar si el servicio brindado es percibido adecuado, de acuerdo a su demanda. Asimismo se redactaron unas recomendaciones generales (ver apéndice 2), respecto de, entre otras cosas, los criterios para la selección de psicólogos y estudiantes de psicología voluntarios, las medidas para el asesoramiento eficaz del trabajo de ellos y las condiciones de atención de pacientes. 


\section{CONCLUSIONES}

- Dictar talleres sobre problemas de interés para la población local, resulta una fórmula efectiva para que los vecinos del distrito se enteren de la existencia del Proyecto y asistan en busca de los servicios allí brindados.

- En concordancia con lo hallado por Miljánovich et al. (2014), se encontraron creencias desadaptativas en hombres y mujeres que justifican la violencia física, ya sea como método de crianza o como patrón de conducta dentro de la relación de pareja.

- Los adolescentes del colegio no escolarizado y del colegio público no se mostraron motivados con el taller y la charla de a las que asistieron. Este hallazgo encuentra sentido en el hecho de que en general a mayor edad, mayor percepción de riesgo y viceversa; independientemente de la cantidad de información que se posea (García del Castillo, López-Sánchez, García y Dias; 2014).

- Gran parte de los pacientes atendidos no regresaba después de la primera consulta, y la mayoría no asistía a más de tres sesiones.

- Otra dificultad con la que hubo que lidiar en el desarrollo de las sesiones de psicoterapia, fue el pensamiento concreto y la capacidad intelectual limitada que caracteriza a la población usuaria de los servicios del Proyecto (aunque con excepciones). Ello sumado a una pobre habilidad para la expresión y comprensión verbal, dificultaban la cabal realización de técnicas cognitivas, el entendimiento de metáforas e incluso la comprensión de los síntomas.

- Ante estas características, intervenciones basadas en el Análisis Funcional del Comportamiento y las técnicas conductistas, resultan la forma más eficiente de abordar los 
motivos de consulta, pudiéndose a la vez, adaptar las estrategias ante pacientes con un mayor potencial intelectual. 


\section{RECOMENDACIONES}

- Desarrollar los talleres futuros de acuerdo a los criterios del diseño instruccional, para contrastar las medidas de las variables de interés pre y post intervención.

- Procurar que los talleres con adolescentes sean desarrollados únicamente con voluntarios que realmente quieran participar en ellos. De este modo, se garantiza que quienes verdaderamente deseen aprender nuevas competencias, no sean distraídos por los que no. A la vez, la medición post intervención reflejará su verdadero impacto y al reducir el número de asistentes, se dará una enseñanza más personalizada, lo que suele redundar en más altos niveles de aprendizaje.

- Modificar el abordaje con adolescentes de aspectos ligados a la prevención en adicciones y conductas sexuales de riesgo, considerando lo señalado por García del Castillo et al. (2014), esto es, que en lugar de centrarse en la transmisión de información, especialmente de las consecuencias y riesgos de tales comportamientos, se transmitan mensajes positivos que refuercen estilos de vida saludables. Lo cual en última instancia supone disertar sobre los beneficios del comportamiento sano, desde un enfoque tipo publicitario: girando en torno a las emociones positivas (amor, felicidad, comodidad, paz, etc.).

- Ejecutar los esfuerzos de orientación vocacional de manera personalizada, evaluando en cada alumno sus aptitudes y sus intereses y proporcionándole una retroalimentación individual, triangulando la información recabada con los datos existentes sobre la oferta educativa y las ayudas estatales acordes con su realidad socioeconómica.

- Adecuar las técnicas terapéuticas de intervención a las características de cada paciente, observando cuidadosamente la evidencia empírica que las avala. 
- Aplicar la encuesta de satisfacción terapéutica (apéndice 1) a cada paciente después de la primera sesión, y en adelante cada cinco sesiones; y analizar los resultados obtenidos.

- Investigar los factores que generan el abandono temprano del proceso terapéutico en gran parte de la población atendida.

- Revisar y analizar las recomendaciones realizadas (apéndice 2) y ejecutar las que se consideren convenientes. 


\section{REFERENCIAS}

Alarcón, R. (2015). Salud Mental y salud Pública en el Perú: ya es tiempo de actuar. Revista de Neuro-Psiquiatría, 78(1) 1-2.

Camacho, L. C. (2014). Terapia cognitivo-conductual: pasado, presente y tendencias futuras. En J. M. Escobar. y M. Uribe. (Ed.), Avances en psiquiatría desde un modelo biopsicosocial (pp. 435-497). Bogotá: Universidad de los Andes.

Del Águila-Escobedo, A. (2015). Violencia y estrés infantil: ¿está en juego nuestro futuro?. Acta Médica Peruana, 32(2), 71-83.

Echevarría, B. y Martínez, M. [Adapt]. (2014). Guía de Orientación Profesional Coordinada. Barcelona: Fundación Bertelsmann.

García del Castillo, J. A., López-Sánchez, C., García, Á., y Dias, P. C. (2014). Análisis de la información en la prevención del consumo de drogas y otras adicciones. Salud y Drogas, 14(1), 5-14. doi: 10.21134/haaj.v14i1.225

Miljánovich, M. A., Huerta, R. E., Campos, E., Torres, S. A., Vásquez, V. A., Vera, K., y Díaz, G. (2014). Violencia familiar: modelos explicativos del proceso a través del estudio de casos. Revista De Investigación En Psicología, 16(1), 29-44.

Pérez, M. (2014). Las terapias de tercera generación como terapias contextuales. Madrid: Síntesis.

Salas, F. G. (2018). Caracterización de factores implicados en las conductas de riesgo en adolescentes. Revista ABRA, 38(56), 1-16. doi: 10.15359/abra.38-56.3

Sánchez, M., Martínez, M., González, C., Pérez, J., González, N., y Martínez, P. (2017). Satisfacción vocacional con la elección del ciclo de formación profesional. Revista de Estudios e Investigación en Psicología y Educación, Extr(7), 36-51. doi: 10.17979/reipe.2017.0.07.2344 


\section{APÉNDICES}




\section{APÉNDICE 1: ECUESTA DE SATISFACCIÓN TERAPÉUTICA}

La siguiente encuesta tiene como fin conocer su grado de satisfacción con los servicios que ha recibido de parte de su terapeuta/consejero. Por ello, se le pide que responda con la mayor sinceridad posible. Tenga presente que la encuesta es anónima. Le agradecemos de antemano por su cooperación.

\section{Nombre del terapeuta/consejero:}

Marque la casilla que corresponda según su grado de satisfacción con lo indicado en cada pregunta. Una marca en la casilla número 1 implicaría nula satisfacción, en la casilla número 5 una satisfacción moderada y en la casilla número 10 una extrema satisfacción. También puede marcar las casillas $2 ; 3 ; 4 ; 6 ; 7 ; 8$ ó 9. Recuerde que a mayor satisfacción, mayor deberá ser el número que marque.

La confianza que siento con mi terapeuta/consejero, me produce una satisfacción de:

\begin{tabular}{|l|l|l|l|l|l|l|l|l|l|}
\hline 1 & 2 & 3 & 4 & 5 & 6 & 7 & 8 & 9 & 10 \\
\hline
\end{tabular}

Las explicaciones que me da mi terapeuta/consejero sobre por qué pasan ciertas cosas y la forma en que entiende mi situación, me producen una satisfacción de:

\begin{tabular}{|l|l|l|l|l|l|l|l|l|l|}
\hline 1 & 2 & 3 & 4 & 5 & 6 & 7 & 8 & 9 & 10 \\
\hline
\end{tabular}


El alivio y la tranquilidad que tengo después de mis sesiones con mi terapeuta/consejero, me producen una satisfacción de:

\begin{tabular}{|l|l|l|l|l|l|l|l|l|l|}
\hline 1 & 2 & 3 & 4 & 5 & 6 & 7 & 8 & 9 & 10 \\
\hline
\end{tabular}

Con las estrategias que me ha brindado mi terapeuta/consejero para solucionar los problemas por los que solicité la consulta, mi grado de satisfacción es de:

\begin{tabular}{|l|l|l|l|l|l|l|l|l|l|}
\hline 1 & 2 & 3 & 4 & 5 & 6 & 7 & 8 & 9 & 10 \\
\hline
\end{tabular}




\section{APÉNDICE 2: RECOMENDACIONES GENERALES}

$\underline{\text { Recomendaciones para la estandarización de los servicios brindados desde el Proyecto }}$

Elaborado por Carlos Miranda

I. En general.

- Tener por escrito las políticas generales del proyecto.

- Comunicarlas y entregarlas a la institución en que se halla.

- Comunicarlas y entregarlas a los nuevos integrantes del equipo.

\section{Para la atención de pacientes.}

- Estipular los motivos de consulta que atendrán los estudiantes (recomendación: todos aquellos para los que cuenten con los conocimientos y/o experiencia necesaria de técnicas empíricamente validadas que permitan reducir los síntomas; determinación que deberán tomar los docentes).

- Estipular los motivos de consulta que atenderán los psicólogos (recomendación: todos aquellos para los que cuenten con los conocimientos y/o experiencia necesaria de técnicas empíricamente validadas que permitan reducir los síntomas; determinación que deberá tomar el propio docente que asuma el caso).

- Estipular los motivos de consulta que no serán atendidos (recomendación: ideación suicida, casos de psicosis sin tratamientos psicofarmacológicos paralelos, casos de bipolaridad sin tratamientos psicofarmacológicos paralelos y todos aquellos casos para los que quien asuma el tratamiento no cuente con los conocimientos y/o experiencia de las técnicas empíricamente validadas que 
permiten aliviar los síntomas emocionales y conductuales; determinación que deberán tomar los docentes en su conjunto).

- Establecer el tiempo límite máximo que deberá durar una sesión (recomendación: 60 minutos).

- Establecer los criterios para la derivación interna (recomendación: compatibilidad de horarios entre terapeuta y paciente, mayores conocimientos y/o experiencia de otro miembro del equipo para generar un mayor alivio terapéutico en el paciente).

- Establecer los criterios para la derivación externa con exclusión del tratamiento en el departamento (recomendación: los mencionados en los motivos de consulta que no se atenderán, y todas aquellas problemáticas para las que exista una oferta de tratamiento especializada identificada, que sea accesible a la capacidad económica del paciente).

- Establecer los criterios para la derivación externa sin exclusión del tratamiento en el departamento (recomendación: trastornos psicóticos con tratamientos psicofarmacológicos paralelos, bipolaridad con tratamientos psicofarmacológicos paralelos, depresión mayor moderada con tratamientos psicofarmacológicos paralelos, casos de trastorno límite de personalidad con conductas impulsivas o autodestructivas con tratamientos psicofarmacológicos paralelos, trastorno paranoide con tratamientos psicofarmacológicos; trastornos neuróticos con somatizaciones, y todos los demás trastornos con los que el paciente pueda adquirir habilidades conductuales y alivios emocionales significativos recibiendo atención psicológica, si recibe un tratamiento psicofarmacológico paralelo, siendo que si no lo recibe, pone en peligro su integridad física en general y su salud psicosocial). 
- Delimitar el tiempo máximo de espera al paciente que no llega puntualmente a su cita (recomendación: 15 minutos).

- Determinar el tiempo de sesión que se le dará al paciente que no llega puntualmente (recomendación: la resta entre el tiempo límite máximo de atención y la cantidad de minutos tarde que llegue. Por ejemplo: 60-10=50 $\min )$.

- Determinar las técnicas que no serán empleadas en el tratamiento de pacientes (recomendación: todas aquellas que no estén empíricamente validadas. Por ejemplo, PNL. Y todas aquellas para las que el terapeuta/consejero no cuente con los conocimientos y/o experiencia de su correcta aplicación).

- Delimitar la máxima cantidad de pacientes que verá un miembro del equipo por día.

\section{Para el asesoramiento de estudiantes}

- Cada psicólogo dedicará una hora a la semana de su tiempo en el departamento para asesorar el abordaje terapéutico de los casos que ven los estudiantes.

- Cada psicólogo dará mensualmente una retroalimentación a los estudiantes sobre su desempeño en el departamento.

- Cada semestre los psicólogos en su conjunto darán una retroalimentación a los estudiantes sobre su desempeño en el departamento.

- Cada estudiante nuevo ingresará a un mínimo número determinado de sesiones como observador antes de abordar solo un caso (recomendación: 4). 
- Cada semestre, todo estudiante será observado por algún psicólogo en un número mínimo de sesiones - pueden ser más, pero debe haber un mínimo(recomendación: 4).

- Cada semestre, todo estudiante ingresará como observador a las sesiones de algún psicólogo en un mínimo de sesiones - pueden ser más, pero debe haber un mínimo- (recomendación: 4).

- Cada estudiante debe reportar al menos a un psicólogo un número mínimo de casos vistos por semana -aunque pueden ser más- (recomendación: 2).

- Los psicólogos deberán supervisar constantemente la evolución de los pacientes cuyos cuadros clínicos sean especialmente delicados.

\section{Para la selección de estudiantes}

- Establecer criterios específicos para seleccionar a los estudiantes, cuando el número de vacantes sea inferior al número de candidatos.

- Las recomendaciones para ello son: revisar la ubicación relativa del alumno dentro de su la facultad (por ejemplo, quinto superior), las notas que ha obtenido en los cursos de psicología clínica, la cantidad de electivos que ha llevado de psicología clínica, el CV del candidato.

- Si la elección entre los candidatos fuera muy complicada, por exhibir éstos un gran potencial de desempeño, aplicar una prueba de conocimientos.

- Establecer el perfil del interno/voluntario. 